CUBO A Mathematical Journal Vol.19, No 03, (01-14). October 2017

\title{
The Solvability and Fractional Optimal Control for Semilinear Stochastic Systems
}

\author{
SuRENDRA KumAR \\ Department of Mathematics, \\ University of Delhi, Delhi-11000\%, \\ India. \\ mathdma@gmail.com
}

\begin{abstract}
This paper deals with fractional optimal control for a class of semilinear stochastic equation in Hilbert space setting. To ensure the existence and uniqueness of mild solution, a set of sufficient conditions is constructed. The existence of fractional optimal control for semilinear stochastic system is also discussed. Finally, an example is included to show the applications of the developed theory.
\end{abstract}

\section{RESUMEN}

Este artículo estudia el control óptimo fraccional para una clase de ecuaciones estocásticas semilineales en un contexto de espacios de Hilbert. Para asegurar la existencia y la unicidad de soluciones blandas, construimos un conjunto de condiciones suficientes. La existencia del control óptimo fraccional para sistemas estocásticos semilineales también es discutido. Finalmente, incluimos un ejemplo para mostrar la aplicabilidad de la teoría desarrollada.

Keywords and Phrases: Fractional calculus, Semilinear stochastic system, Mild solution, Optimal control, Fixed point theory

2010 AMS Mathematics Subject Classification: 26A33, 34A08, 34A12, 47H10, 49J99, 93C23 


\section{Introduction}

Fractional calculus is about to generalization of the integer order integral and derivative to arbitrary order. The potential applications of fractional calculus are in many fields of science and engineering including fluid flow, electrical networks, diffusive transport, rheology, control theory, electromagnetic theory, and probability [13, 10, 9, 5, 7, 8, 15. It is well known that many real world problems in science and engineering are modeled as stochastic differential equations [4. Since fractional stochastic differential equations describe a physical dynamical system more accurately, it seems necessary to discuss the qualitative properties for such systems.

If a fractional differential equation describes the performance index and system dynamics, then an optimal control problem is known as a fractional optimal control problem. Using the fractional variational principle and Lagrange multiplier technique, Agrawal [1] discussed the general formulation and solution scheme for Riemann-Liouville fractional optimal control problems. It is remarkable thathe fixed point technique, which is used to establish the existence result for abstract fractional differential equations, could be extended to address the fractional optimal control problems. Wang et. al. [19, 20] discussed the existence of local and global solutions for fractional semilinear systems, and extended the results for fractional optimal control. Using fractional powers of the linear operator, the existence of fractional optimal controls for the Lagrange problem investigated in infinite dimensional reflexive Banach space [21, 22, 24]. Wang et al. 23] studied the solvability and the existence of optimal controls for fractional integro-differential systems with infinite delay via contraction mapping principle. Pan et al. [14 constructed a set of sufficient conditions that guarantees the existence of optimal control to the Riemann-Liouville fractional control systems in the Banach space. Li and Liu [11] presented the optimal control for nonlinear impulsive differential equations in Banach space setting. Yan and $\mathrm{Lu}$ [25] investigated the optimal control problems for fractional stochastic neutral integro-differential equations with infinite delay in a Hilbert space by using the fractional calculus, stochastic analysis theory, and fixed point theorem.

Using the LeraySchauder fixed point theorem, Balasubramaniam and Tamilalagan [2] studied the solvability and optimal controls for impulsive fractional stochastic integro-differential equations. Recently, Tamilalagan and Balasubramaniam [18] investigated the solvability and optimal controls for fractional stochastic differential equations driven by Poisson jumps in Hilbert space by using analytic resolvent operators and classical Banach contraction mapping principle.

By motivated from the above work, the main objective of this paper is to obtain sufficient conditions for existence and uniqueness of mild solution of fractional stochastic semilinear system of fractional order via contraction mapping principle. To prove the existence and uniqueness of mild solution it is assumed that nonlinear functions satisfying Lipschitz continuity and linear growth conditions. Next, we introduce a formulation for fractional optimal control governed by the fractional stochastic semilinear systems, where the fractional derivative is defined in the sense of the Caputo. Finally, The existence of fractional optimal controls for the Lagrange problem 
is investigated. The obtained results are new and considered as a contribution to the theory of stochastic fractional optimal control.

The rest of the paper is organized as follows: in Sect. 2] we present some basic definitions, notations and lemmas as preliminaries. In Sect. 3, the existence and uniqueness of mild solution are proved. Existence of fractional optimal control is shown in Sect. 4. In Sect. 5, an example is given to illustrate the theory.

\section{Preliminaries}

This section contains basic definitions, notations and preliminary results, which help us to obtain desired results. Throughout this paper, we use the following notations: Let $\mathrm{H}$ and $\mathrm{K}$ be separable Hilbert spaces. For convenience, we denote the inner products and norms in all spaces by $\langle\cdot, \cdot\rangle$ and $\|\cdot\|$, respectively. Let $(\Omega, \mathcal{F}, \mathbf{P})$ be a complete probability space furnished with complete family of right continuous increasing sub $\sigma$-algebras $\left\{\mathcal{F}_{\mathrm{t}}: 0 \leq \mathrm{t} \leq \tau\right\}$ satisfying $\mathcal{F}_{\mathrm{t}} \subset \mathcal{F}$. Let $\left\{\boldsymbol{e}_{\mathrm{n}}\right\}_{\mathfrak{n}=1}^{\infty}$ be a complete orthonormal system in $K$, and $\left\{\beta_{n}\right\}_{n=1}^{\infty}$ a sequence of independent Brownian motions such that

$$
\omega(t):=\sum_{n=1}^{\infty} \sqrt{\lambda_{n}} e_{n} \beta_{n}(t), t \in[0, \tau]
$$

where $\left\{\lambda_{n}\right\}_{n=1}^{\infty}$ is a bounded sequence of non-negative real numbers such that $Q e_{n}=\lambda_{n} e_{n}, n=$ $1,2, \cdots$ with $\operatorname{tr}(\mathrm{Q})=\sum_{n=1}^{\infty} \lambda_{n}<\infty(\operatorname{tr}(\mathrm{Q})$ denotes the trace of the operator $\mathrm{Q})$. Then the above $\mathrm{K}$-valued stochastic process $\omega(\mathrm{t})$ is called a Wiener process. The normal filtration $\mathcal{F}_{\mathrm{t}}$ is the sigma algebra generated by $\{\omega(\mathrm{s}): 0 \leq \mathrm{s} \leq \mathrm{t}\}$ and $\mathcal{F}_{\tau}=\mathcal{F}$.

Denoted by $\mathrm{L}(\mathrm{K}, \mathrm{H})$ the space of all bounded operators from $\mathrm{K}$ into $\mathrm{H}$ equipped with the usual operator norm. For $\psi \in \mathrm{L}(\mathrm{K}, \mathrm{H})$, we define

$$
\|\psi\|_{\mathrm{Q}}^{2}=\operatorname{tr}\left(\psi \mathrm{Q} \psi^{*}\right)=\sum_{n=1}^{\infty}\left\|\sqrt{\lambda_{\mathrm{n}}} \psi e_{\mathrm{n}}\right\|^{2}
$$

If $\|\psi\|_{\mathrm{Q}}^{2}<\infty$, then $\psi$ is called a Q-Hilbert Schmidt operator. Let $\mathrm{L}_{\mathrm{Q}}(\mathrm{K}, \mathrm{H})$ be the space of all Q-Hilbert Schmidt operators $\psi: K \rightarrow H$. The completion $\mathrm{L}_{\mathrm{Q}}(\mathrm{K}, \mathrm{H})$ of $\mathrm{E}(\mathrm{K}, \mathrm{H})$ with respect to the topology induced by the norm $\|\cdot\|_{\mathrm{Q}}$, where $\|\psi\|_{\mathrm{Q}}^{2}=\langle\psi, \psi\rangle$ is a Hilbert space with the above norm topology.

The space of strongly measurable, $\mathrm{H}$-valued, square integrable random variables, denoted by $\mathrm{L}_{2}(\Omega, \mathrm{H})$, is a Banach space equipped with the norm topology $\|x(\cdot)\|=\left(\mathbb{E}\|x(t)\|^{2}\right)^{1 / 2}$, where $\mathbb{E}(\cdot)$ is the expectation with respect to the measure $\mathbf{P}$.

Let $C\left([0, \tau], L_{2}(\Omega, H)\right)$ be the Banach space of continuous maps from $[0, \tau]$ into $L_{2}(\Omega, H)$ satisfying $\sup _{0 \leq t \leq \tau} \mathbb{E}\|x(t)\|^{2}<\infty$. Let $H_{2}$ be the closed subspace of $C\left([0, \tau], L_{2}(\Omega, H)\right)$ consisting 
of measurable, $\mathcal{F}_{t}$-adapted, $H$-valued processes $x \in C\left([0, \tau], L_{2}(\Omega, H)\right)$ equipped with the norm

$$
\|x\|_{H_{2}}:=\left(\sup _{0 \leq t \leq \tau} \mathbb{E}\|x(t)\|^{2}\right)^{1 / 2}
$$

Consider the following integral cost functional

$$
J(x, u):=\mathbb{E}\left\{\int_{0}^{\tau} \ell\left(t, x^{u}(t), u(t)\right) d t\right\}
$$

subject to the state equation

$$
\left.\begin{array}{l}
{ }^{C} D_{t}^{\alpha} x(t)=A x(t)+B(t) u(t)+f(t, x(t))+g(t, x(t)) \frac{d \omega(t)}{d t}, \\
x(0)=x_{0} \in H,
\end{array}\right\}
$$

where the integrand $\ell:[0, \tau] \times \mathrm{H} \times \mathrm{U} \rightarrow \mathbb{R} \cup\{\infty\}$ is specified latter; ${ }^{C} \mathrm{D}_{\mathrm{t}}^{\alpha}$ is the Caputo fractional derivative of order $0<\alpha<1$, the state $x(\cdot)$ is $\mathrm{H}$-valued stochastic process; the control function $u(\cdot)$ takes its values from a separable reflexive Hilbert space $\mathrm{U} ; A: D(A) \subseteq H \rightarrow H$ is the infinitesimal generator of a resolvent $S_{\alpha}(t), t \geq 0$ on $H ;\{B(t): t \geq 0\}$ is a family of linear operators from $U$ to $\mathrm{H}$; the functions $\mathrm{f}:[0, \tau] \times \mathrm{H} \rightarrow \mathrm{H}$ and $\mathrm{g}:[0, \tau] \times \mathrm{H} \rightarrow \mathrm{L}(\mathrm{K}, \mathrm{H})$ are nonlinear, $\mathrm{L}(\mathrm{K}, \mathrm{H})$ denotes the space of all bounded linear operators form $E$ into $H, x_{0}$ is $\mathcal{F}_{0}$-measurable $\mathrm{H}$-valued random variable independent of $\omega$.

Define the admissible set $\mathrm{U}_{\mathrm{ad}}$, the set of all $v(\cdot):[0, \tau] \times \Omega \rightarrow \mathrm{U}$ such that $v$ is $\mathcal{F}_{\mathrm{t}}$ adapted stochastic process and $\mathbb{E} \int_{0}^{\tau}\|v(t)\|^{p} \mathrm{dt}<\infty$. Clearly $\mathrm{U}_{\mathrm{ad}} \neq \emptyset$ and $\mathrm{U}_{\mathrm{ad}} \subset \mathrm{L}^{\mathrm{p}}([0, \tau] ; \mathrm{U})(1<\mathrm{p}<$ $+\infty)$ is bounded, closed, and convex.

Denoted by the set of all admissible state-control pairs $(x, u)$ by $\mathcal{A}_{\mathrm{ad}}$, where $\mathrm{x}$ is the mild solution of the system (2.2) corresponding to the control $u \in \mathrm{U}_{\mathrm{ad}}$. The main objective of this paper is to find a pair $\left(x^{0}, u^{0}\right) \in \mathcal{A}_{\mathrm{ad}}$ such that

$$
J\left(x^{0}, u^{0}\right):=\inf \left\{J(x, u):(x, u) \in \mathcal{A}_{\mathrm{ad}}\right\}=\varepsilon .
$$

We now recall the following well known definitions related to fractional order differentiation and integration. For more details on fractional calculus one can see [13, 10.

Definition 2.1 The Riemann-Liouville fractional integral operator of order $\alpha>0$ of a function $f:[0, \infty) \rightarrow \mathbb{R}$ with the lower limit 0 is defined as

$$
I^{\alpha} f(t)=\frac{1}{\Gamma(\alpha)} \int_{0}^{t}(t-s)^{\alpha-1} f(s) d s
$$

where $\Gamma$ is the Euler gamma function.

Definition 2.2 The Caputo fractional derivative of order $\alpha>0$ for the function $f \in C^{m}([0, \tau], \mathbb{R})$ is defined by

$$
{ }^{c} D_{t}^{\alpha} f(t)=\frac{1}{\Gamma(m-\alpha)} \int_{0}^{t}(t-s)^{m-\alpha-1} f^{(m)}(s) d s, \quad m-1<\alpha<m \in \mathbb{N} .
$$


If $f$ is an abstract function with values in $H$, then the integrals appearing in Definition 2.1 and Definition 2.2 are taken in the Bochner sense. Moreover, the Caputo derivative of a constant is always zero.

The two-parameter function of the Mittag-Leffler type is defined by the series expansion

$$
\mathrm{E}_{\alpha, \beta}(z)=\sum_{j=1}^{\infty} \frac{z^{j}}{\Gamma(\alpha j+\beta)}=\frac{1}{2 \pi i} \int_{C} e^{\lambda} \frac{\lambda^{\alpha-\beta}}{\lambda^{\alpha}-z} \mathrm{~d} \lambda ; \alpha, \beta>0, z \in \mathbb{C},
$$

where $C$ is a contour that start and end at $-\infty$ and encircles the disc $\|\lambda\| \leq|z|^{1 / 2}$ counterclockwise (see [6] for more results on Mittag-Leffler function).

Definition 2.3 [16, 17] A closed and linear operator $A$ is said to be sectorial of type $\mu$ if there exist $\pi / 2 \leq \theta \leq \pi, \tilde{M}>0$ and $\mu \in \mathbb{R}$ such that the following conditions are satisfied: $\rho(A) \subset$ $\Sigma_{(\theta, \mu)}:=\{\lambda \in \mathbb{C}: \lambda \neq \mu,|\arg (\lambda-\mu)|<\theta\}$, and $\|R(\lambda, A)\|:=\left\|(\lambda-A)^{-1}\right\| \leq \frac{\tilde{M}}{|\lambda-\mu|}, \lambda \in \Sigma_{(\theta, \mu)}$.

Lemma 2.1 [17] For $0<\alpha<2$, a linear closed densely defined operator $A$ belongs to $A^{\alpha}\left(\theta_{0}, \mu_{0}\right)$ if and only if $\lambda^{\alpha} \in \rho(A)$ for each $\lambda \in \Sigma_{\left(\theta_{0}+\pi / 2, \mu\right)}$ and for any $\mu>\mu_{0}, \theta<\theta_{0}$ there is a constant $\mathrm{C}_{0}=\mathrm{C}_{0}(\theta, \mu)$ such that

$$
\left\|\lambda^{\alpha-1} R\left(\lambda^{\alpha}, A\right)\right\| \leq \frac{C_{0}}{|\lambda-\mu|}, \text { for } \lambda \in \Sigma_{(\theta+\pi / 2, \mu)} .
$$

Lemma 2.2 [17] If $F$ satisfies the uniform Hölder condition with the exponent $0<\gamma \leq 1$ and $A$ is a sectorial operator, then the unique solution of the Cauchy problem

$$
\left.\begin{array}{l}
{ }^{c} D_{t}^{\alpha} y(t)=A y(t)+F(t), \quad 0<\alpha<1, \quad t \in(0, \tau], \\
y(0)=y_{0},
\end{array}\right\}
$$

is given by

$$
y(t)=S_{\alpha}(t) y_{0}+\int_{0}^{t} T_{\alpha}(t-s) F(s) d s
$$

where

$$
\begin{aligned}
& S_{\alpha}(t)=E_{\alpha, 1}\left(A t^{\alpha}\right)=\frac{1}{2 \pi i} \int_{\widehat{B}_{\rho}} e^{\lambda t} \frac{\lambda^{\alpha-1}}{\lambda^{\alpha}-A} d \lambda, \\
& T_{\alpha}(t)=t^{\alpha-1} E_{\alpha, \alpha}\left(A t^{\alpha}\right)=\frac{1}{2 \pi i} \int_{\widehat{B}_{\rho}} e^{\lambda t} \frac{1}{\lambda^{\alpha}-A} d \lambda,
\end{aligned}
$$

$\widehat{B}_{\rho}$ is the Bromwich path, $T_{\alpha}(t)$ is called the $\alpha$-resolvent family, and $S_{\alpha}(t)$ is the solution operator generated by $A$.

An operator $A$ is said to belong to $\mathfrak{C}^{\alpha}(\tilde{M}, \mu)$ if problem (2.3) with $F=0$ has a solution operator $S_{\alpha}(\mathrm{t})$ satisfying $\left\|S_{\alpha}(\mathrm{t})\right\| \leq \tilde{M} e^{\mu t}$. Denote $\mathfrak{C}^{\alpha}(\mu):=\cup\left\{\mathfrak{C}^{\alpha}(\tilde{M}, \mu): \tilde{M} \geq 1\right\}, \mathfrak{C}^{\alpha}:=\left\{\mathfrak{C}^{\alpha}(\mu): \mu \geq 0\right\}$, and $\mathcal{A}^{\alpha}\left(\theta_{0}, \mu_{0}\right)=\left\{A \in \mathfrak{C}^{\alpha}:\right.$ A generates analytic solution operators $S_{\alpha}(t)$ of type $\left.\left(\theta_{0}, \mu_{0}\right)\right\}$. 
If $0<\alpha<1$ and $A \in \mathcal{A}^{\alpha}\left(\theta_{0}, \mu_{0}\right)$, then we have $\left\|S_{\alpha}(t)\right\| \leq \tilde{M} e^{\mu t}$ and $\left\|T_{\alpha}(t)\right\| \leq C e^{\mu t}(1+$ $\left.t^{\alpha-1}\right), t>0, \mu>\mu_{0}$. If

$$
M_{S}:=\sup _{0 \leq t \leq \tau}\left\|S_{\alpha}(t)\right\|, \quad M_{T}:=\sup _{0 \leq t \leq \tau} C e^{\mu t}\left(1+t^{1-\alpha}\right),
$$

then, we have

$$
\left\|S_{\alpha}(t)\right\| \leq M_{S}, \quad\left\|T_{\alpha}(t)\right\| \leq t^{\alpha-1} M_{T} .
$$

By Lemma 2.2, a mild solution of the system (2.2) is defined as

Definition 2.4 An $\mathcal{F}_{\mathrm{t}}$-adapted stochastic process $x(\mathrm{t}) \in \mathrm{C}\left([0, \tau], \mathrm{L}^{2}(\Omega, \mathcal{F}, \mathrm{H})\right.$ is called a mild solution of system (2.2) if for each $u(\cdot) \in \mathrm{L}^{\mathrm{p}}([0, \tau] ; \mathrm{U}), x(\mathrm{t})$ is measurable and the following stochastic integral equation is satisfied:

$$
\begin{aligned}
x(t)= & s_{\alpha}(t) x_{0}+\int_{0}^{t} T_{\alpha}(t-s)[B(s) u(s)+f(s, x(s))] d s \\
& +\int_{0}^{t} T_{\alpha}(t-s) g(s, x(s)) d \omega(s) .
\end{aligned}
$$

Lemma 2.3[12] A measurable function $\chi:[0, \tau] \rightarrow \mathrm{V}$ is Bochner integrable, if $\|x\|$ is Lebesgue integrable.

\section{Existence and Uniqueness of Mild Solution}

To prove the existence and uniqueness of mild solution of the system (2.2), we impose the following conditions to the system parameter:

[H0] For any $x \in H$, the function $t \rightarrow f(t, x(t))$ and $t \rightarrow g(t, x(t))$ are $\mathcal{F}_{t}$-measurable.

[H1] The functions $f:[0, \tau] \times H \rightarrow H, g:[0, \tau] \times H \rightarrow E(K, H)$ are continuous, and satisfying linear growth and Lipschitz conditions. Moreover, without loss of generality, we may assume that there are positive constants $L_{f}$ and $L_{g}$ such that

$$
\begin{aligned}
& \|f(t, x)-f(t, y)\|^{2} \leq L_{f}\|x-y\|^{2}, \quad\|f(t, x)\|^{2} \leq L_{f}\left(1+\|x\|^{2}\right), \\
& \|g(t, x)-g(t, y)\|_{Q}^{2} \leq L_{g}\|x-y\|^{2}, \quad\|g(t, x)\|_{Q}^{2} \leq L_{g}\left(1+\|x\|^{2}\right) \text {. }
\end{aligned}
$$

[H3] The operator $\mathrm{B} \in \mathrm{L}_{\infty}([0, \tau] ; \mathrm{E}(\mathrm{U}, \mathrm{H}))$ and $\|\mathrm{B}\|_{\infty}$ stand for the norm of operator $\mathrm{B}$ in the Banach space $\mathrm{L}_{\infty}([0, \tau] ; \mathrm{E}(\mathrm{U}, \mathrm{H}))$.

[H4] The multi-valued map $\mathcal{U}(\cdot):[0, \tau] \rightrightarrows 2^{\mathrm{U}} \backslash\{\emptyset\}$ has closed, convex and bounded values; $\mathcal{U}(\cdot)$ is graph measurable and $\mathcal{U}(\cdot) \subseteq \Xi$, where $\Xi$ is a bounded subset of $\mathrm{U}$.

First we show that the system (2.4) has at least one solution.

Theorem 3.1 Under assumptions [H0]-[H3] the system (2.4) admits a unique mild solution on $[0, \tau]$ for each control function $u(\cdot) \in \mathrm{U}_{\mathrm{ad}}$ and for some $p$ such that $p \alpha>1$. 
Proof. Define an operator $\mathrm{Im}: \mathrm{H}_{2} \rightarrow \mathrm{H}_{2}$ as

$$
\begin{aligned}
(\operatorname{Im} x)(t)= & S_{\alpha}(t) x_{0}+\int_{0}^{t} T_{\alpha}(t-s)[B(s) u(s)+f(s, x(s))] d s \\
& +\int_{0}^{t} T_{\alpha}(t-s) g(s, x(s)) d \omega(s) .
\end{aligned}
$$

To show that (2.4) is the mild solution of the system (2.4) on $[0, \tau]$, it is enough to prove that Im has a fixed point in the space $\mathrm{H}_{2}$. For this purpose, we will employ the classical fixed point theorem for contractions.

We first show that $\operatorname{Im}\left(\mathrm{H}_{2}\right) \subset \mathrm{H}_{2}$. Let $x \in \mathrm{H}_{2}$, then we have

$$
\mathbb{E}\|(\operatorname{Im} x)(t)\|^{2} \leq 4\left[I_{0}+I_{1}+I_{2}+I_{3}\right]
$$

Clearly $I_{0} \leq M_{S}^{2} \mathbb{E}\left\|x_{0}\right\|^{2}$. Next, using the Cauchy-Schwartz inequality, we have

$$
\begin{aligned}
I_{1} & =\left\|\int_{0}^{t} T_{\alpha}(t-s) B(s) u(s) d s\right\|^{2} \\
& \leq M_{T}^{2}\|B\|_{\infty}^{2}\left[\int_{0}^{t}(t-s)^{\alpha-1}\|u(s)\| d s\right]^{2} \\
& \leq M_{T}^{2}\|B\|_{\infty}^{2}\left[\left(\int_{0}^{t}(t-s)^{\frac{p(\alpha-1)}{p-1}} d s\right)^{\frac{p-1}{p}}\left(\int_{0}^{t}\|u(s)\|_{u}^{p} d s\right)^{\frac{1}{p}}\right]^{2} \\
& \leq M_{T}^{2}\|B\|_{\infty}^{2}\|u\|_{L^{p}([0, \tau] ; u)}^{2} \tau^{2\left(\frac{p \alpha-1}{p}\right)}\left(\frac{p-1}{p \alpha-1}\right)^{\frac{2(p-1)}{p}}
\end{aligned}
$$

The Cauchy-Schwartz inequality, and hypothesis (H1) imply that

$$
\begin{aligned}
\mathrm{I}_{2} & =\mathbb{E}\left\|\int_{0}^{t} \mathrm{~T}_{\alpha}(\mathrm{t}-\mathrm{s}) \mathbb{E} f(s, x(s)) \mathrm{d} s\right\|^{2} \\
& \leq\left(\int_{0}^{t}\left\|\mathrm{~T}_{\alpha}(\mathrm{t}-\mathrm{s})\right\| \mathbb{E}\|f(s, x(s))\| \mathrm{ds}\right)^{2} \\
& \leq\left(\int_{0}^{t} M_{\mathrm{T}}(\mathrm{t}-\mathrm{s})^{\frac{\alpha-1}{2}}(t-s)^{\frac{\alpha-1}{2}} \mathbb{E}\|f(s, x(s))\| \mathrm{ds}\right)^{2} \\
& \leq M_{\mathrm{T}}^{2}\left(\int_{0}^{t}(t-s)^{\alpha-1} \mathrm{ds}\right)\left(\int_{0}^{t}(t-s)^{\alpha-1}\|\mathbb{E}\| f(s, x(s)) \|^{2} \mathrm{ds}\right) \\
& \leq M_{\mathrm{T}}^{2} \mathrm{~L}_{\mathrm{f}} \frac{\tau^{\alpha}}{\alpha} \int_{0}^{t}(t-s)^{\alpha-1}\left(1+\mathbb{E}\|x(s)\|^{2}\right) \mathrm{d} s \\
& \leq M_{\mathrm{T}}^{2} \mathrm{~L}_{\mathrm{f}} \frac{\tau^{2 \alpha}}{\alpha^{2}}\left(1+\|x\|_{\mathrm{H}_{2}}^{2}\right)
\end{aligned}
$$


and

$$
\begin{aligned}
I_{3} & =\mathbb{E}\left\|\int_{0}^{t} T_{\alpha}(t-s) \mathbb{E} g(s, x(s)) d \omega(s)\right\|^{2} \\
& \leq M_{T}^{2} \operatorname{tr}(Q)\left(\int_{0}^{t}(t-s)^{\alpha-1} d s\right)\left(\int_{0}^{t}(t-s)^{\alpha-1} \mathbb{E}\|g(s, x(s))\|_{Q}^{2} d s\right) \\
& \leq M_{T}^{2} \operatorname{tr}(Q) L_{g} \frac{\tau^{2 \alpha}}{\alpha^{2}}\left(1+\|x\|_{H_{2}}^{2}\right) .
\end{aligned}
$$

Thus 3.1 becomes

$$
\mathbb{E}\|(\operatorname{Im} x)(t)\|^{2} \leq a+b\|x\|_{\mathrm{H}_{2}}^{2},
$$

where $a$ and $b$ are suitable positive constants. This implies that $\mathrm{Im}$ map $\mathrm{H}_{2}$ into itself.

Next, we show that Im is a contraction map. For $x, y \in \mathrm{H}_{2}$, the Cauchy-Schwartz inequality, and hypothesis (H1) yield that

$$
\begin{aligned}
\mathbb{E}\|(\operatorname{Im} x)(t)-(\operatorname{Im} y)(t)\|^{2} \leq & 2 \mathbb{E}\left\|\int_{0}^{t} T_{\alpha}(t-s)[f(s, x(s))-f(s, y(s))] d s\right\|^{2} \\
& +2 \mathbb{E}\left\|\int_{0}^{t} T_{\alpha}(t-s)[g(s, x(s))-g(s, y(s))] d \omega(s)\right\|^{2} \\
\leq & 2 M_{T}^{2}\left(L_{f}+L_{g} \operatorname{tr}(Q)\right) \frac{\tau^{2 \alpha}}{\alpha^{2}}\|x-y\|_{H_{2}}^{2} .
\end{aligned}
$$

Consequently if

$$
2 M_{T}^{2}\left(L_{f}+L_{g} \operatorname{tr}(Q)\right) \frac{\tau^{2 \alpha}}{\alpha^{2}}<1,
$$

then the operator Im has a unique fixed point in $\mathrm{H}_{2}$, which is a solution of the system (2.2). The extra condition on $\tau$ can be easily removed by considering the equation on intervals $[0, \tilde{\tau}],[\tilde{\tau}, 2 \tilde{\tau}]$, $\cdots$ with $\tilde{\tau}$ satisfying (3.2).

We now obtain a priori estimate of mild solution for the system (2.2), that helps us to obtain our main result.

Lemma 3.1 (A priori estimate). Assuming that system (2.4) is the mild solution of system (2.2) on $[0, \tau]$ corresponding to the control $u$. Then there exists a constant $M>0$ such that

$$
E\|x(t)\|^{2} \leq M, \quad \forall t \in[0, \tau] .
$$


Proof. Using condition [H1] and Hölder's inequality, we obtain

$$
\begin{aligned}
\mathbb{E}\|x(t)\|^{2} \leq & 4 \mathbb{E}\left\|S_{\alpha}(t) x_{0}\right\|^{2}+4 \mathbb{E}\left\|\int_{0}^{t} T_{\alpha}(t-s) B(s) u(s) d s\right\|^{2} \\
& +4 \mathbb{E}\left\|\int_{0}^{t} T_{\alpha}(t-s) f(s, x(s)) d s\right\|^{2}+4 \mathbb{E}\left\|\int_{0}^{t} T_{\alpha}(t-s) g(s, x(s)) d \omega(s)\right\|^{2} \\
\leq & 4 M_{S}^{2}\left\|x_{0}\right\|^{2}+4 M_{T}^{2}\|B\|_{\infty}^{2}\left[\int_{0}^{t}(t-s)^{\alpha-1}\|u(s)\| d s\right]^{2} \\
& +4 M_{T}^{2}\left(L_{f}+L_{g} \operatorname{tr}(Q)\right)\left(\int_{0}^{t}(t-s)^{\alpha-1} d s\right) \int_{0}^{t}(t-s)^{\alpha-1}\left\{1+\mathbb{E}\|x(s)\|^{2}\right\} d s \\
\leq & 4 M_{S}^{2}\left\|x_{0}\right\|^{2}+4 M_{T}^{2}\|B\|_{\infty}^{2}\left[\left(\int_{0}^{t}(t-s)^{\frac{p(\alpha-1)}{p-1}} d s\right)^{\frac{p-1}{p}}\left(\int_{0}^{t}\|u(s)\|_{u}^{p} d s\right)^{\frac{1}{p}}\right]^{2} \\
& +4 M_{T}^{2}\left(L_{f}+L_{g} \operatorname{tr}(Q)\right) \frac{\tau^{\alpha}}{\alpha} \int_{0}^{t}(t-s)^{\alpha-1}\left\{1+\mathbb{E}\|x(s)\|^{2}\right\} d s \\
\leq & 4 M_{S}^{2}\left\|x_{0}\right\|^{2}+4 M_{T}^{2}\|B\|_{\infty}^{2}\|u\|_{L^{p}([0, \tau] ; u)}^{2} \tau^{2\left(\frac{p \alpha-1}{p}\right)}\left(\frac{p-1}{p \alpha-1}\right) \\
& +4 M_{T}^{2}\left(L_{f}+L_{g} \operatorname{tr}(Q)\right) \frac{\tau^{2 \alpha}}{\alpha^{2}}+4 M_{T}^{2}\left(L_{f}+L_{g} \operatorname{tr}(Q)\right) \frac{\tau^{\alpha}}{\alpha} \int_{0}^{t}(t-s)^{\alpha-1} \mathbb{E}\|x(s)\|^{2} d s .
\end{aligned}
$$

Now using the Gronwall inequality, one can easily obtain the boundedness of $x$ in $\mathrm{H}_{2}$.

\section{Existence of Fractional Optimal Control}

In this section, we prove the existence of fractional optimal control under the hypothesis:

[HL] Following conditions are imposed on the integrand

$$
\ell:[0, \tau] \times \mathrm{H} \times \mathrm{U} \rightarrow \mathbb{R} \cup\{\infty\}
$$

such that

(i) The integrand $\ell:[0, \tau] \times \mathrm{H} \times \mathrm{U} \rightarrow \mathbb{R} \cup\{\infty\}$ is $\mathcal{F}_{\mathrm{t}}$-measurable.

(ii) The integrand $\ell(t, \cdot, \cdot)$ is sequentially lower semicontinuous on $\mathrm{H} \times \mathrm{U}$ for almost all $\mathrm{t} \in[0, \tau]$;

(iii) The integrand $\ell(t, x, \cdot)$ is convex on $U$ for each $x \in H$ and almost all $t \in[0, \tau]$.

(iv) There exist constants $d \geq 0, e>0, \mu_{0}$ is nonnegative and $\mu_{0} \in \mathrm{L}^{1}([0, \tau] ; \mathbb{R})$ such that

$$
\mu_{0}(t)+d \mathbb{E}\|x\|^{2}+e \mathbb{E}\|u\|_{u}^{p} \leq \ell(t, x, u) .
$$


Theorem 4.1 Suppose hypothesis of Theorem 3.1 and [HL] hold, then Lagrange problem (2.1) admits at least one optimal pair, that is, there exists an admissible state-control pair $\left(x^{0}, u^{0}\right) \in \mathcal{A}_{\text {ad }}$ such that

$$
J\left(x^{0}, u^{0}\right):=\mathbb{E}\left\{\int_{0}^{\tau} \ell\left(t, x^{0}(t), u^{0}(t)\right) d t\right\} \leq J(x, u), \quad \forall(x, u) \in \mathcal{A}_{a d} .
$$

Proof. If $\inf \left\{(x, u) \mid(x, u) \in \mathcal{A}_{\mathrm{ad}}\right\}=+\infty$, then there is nothing to prove. Without any loss of generality, we may assume that $\inf \left\{J(x, u) \mid(x, u) \in \mathcal{A}_{\mathrm{ad}}\right\}=\varepsilon<+\infty$. Now assumption [HL] implies that $\varepsilon>-\infty$. By definition of infimum, there is a minimizing sequence of feasible pairs $\left(x^{m}, u^{m}\right) \in$ $\mathcal{A}_{\mathrm{ad}}$, such that $\mathrm{J}\left(\mathrm{x}^{\mathrm{m}}, \mathrm{u}^{\mathrm{m}}\right) \rightarrow \varepsilon$ as $\mathrm{m} \rightarrow+\infty$. Since $\left\{\mathrm{u}^{\mathrm{m}}\right\} \subseteq \mathrm{U}_{\mathrm{ad}}, \mathrm{m}=1,2, \cdots,\left\{\mathrm{u}^{\mathrm{m}}\right\}$ is a bounded subset of the separable reflexive Banach space $\mathrm{L}^{\mathrm{p}}([0, \tau] ; \mathrm{U})$, there exists a subsequence, relabeled as $\left\{u^{\mathrm{m}}\right\}$ and $u^{0} \in \mathrm{L}^{\mathfrak{p}}([0, \tau] ; U)$ such that $u^{\mathrm{m}} \stackrel{w}{\rightarrow} u^{0}\left(u^{\mathrm{m}} \rightarrow u^{0}\right.$ weakly as $\left.\mathrm{m} \rightarrow+\infty\right)$ in $\mathrm{L}^{\mathfrak{p}}([0, \tau] ; \mathrm{U})$. Since $\mathrm{U}_{\mathrm{ad}}$ is closed and convex, the Mazur lemma forces us to conclude that $u^{0} \in \mathrm{U}_{\mathrm{ad}}$.

Let $\left\{x^{m}\right\}$ be the sequence of solutions of the system (2.2) corresponding to $\left\{u^{m}\right\}$, that is

$$
\begin{aligned}
x^{m}(t):= & S_{\alpha}(t) x_{0}+\int_{0}^{t} T_{\alpha}(t-s)\left[B(s) u^{m}(s)+f\left(s, x^{m}(s)\right)\right] d s \\
& +\int_{0}^{t} T_{\alpha}(t-s) g\left(s, x^{m}(s)\right) d \omega(s) .
\end{aligned}
$$

By Lemma 3.1, it is easy to see that there exists $\delta>0$ such that

$$
\mathbb{E}\left\|x^{m}\right\|^{2} \leq \delta, \quad m=0,1,2, \cdots,
$$

where $x^{0}$ is the mild solution of the system (2.2) corresponding to the control $u^{0} \in \mathrm{U}_{\mathrm{ad}}$ given by

$$
\begin{aligned}
x^{0}(t):= & S_{\alpha}(t) x_{0}+\int_{0}^{t} T_{\alpha}(t-s)\left[B(s) u^{0}(s)+f\left(s, x^{0}(s)\right)\right] d s \\
& +\int_{0}^{t} T_{\alpha}(t-s) g\left(s, x^{0}(s)\right) d \omega(s) .
\end{aligned}
$$

For all $\mathrm{t} \in[0, \tau]$, using condition [H1], the Cauchy-Schwartz inequality and the Hölder inequality, we obtain

$$
\begin{aligned}
& \mathbb{E}\left\|x^{m}(t)-x^{0}(t)\right\|^{2} \leq 3 \mathbb{E}\left\|\int_{0}^{t} T_{\alpha}(t-s)\left[B(s) u^{m}(s)-B(s) u^{0}(s)\right] d s\right\|^{2} \\
& +3 \mathbb{E}\left\|\int_{0}^{t} T_{\alpha}(t-s)\left[f\left(s, x^{m}(s)\right)-f\left(s, x^{0}(s)\right)\right] d s\right\|^{2} \\
& +3 \mathbb{E}\left\|\int_{0}^{t} T_{\alpha}(t-s)\left[g\left(s, x^{m}(s)\right)-g\left(s, x^{0}(s)\right)\right] d \omega(s)\right\|^{2} \\
& \leq 3 M_{T}^{2}\left(\frac{p-1}{p \alpha-1}\right)^{\frac{2 p-2}{p}} \tau^{2 \alpha-\frac{2}{p}}\left(\int_{0}^{t}\left\|B(s) u^{m}(s)-B(s) u^{0}(s)\right\|^{p} d s\right)^{\frac{2}{p}} \\
& +3 M_{\mathrm{T}}^{2} \frac{\tau^{\alpha}}{\alpha}\left(\mathrm{L}_{\mathrm{f}}+\mathrm{L}_{\mathrm{g}} \operatorname{tr}(\mathrm{Q})\right) \int_{0}^{\mathrm{t}}(\mathrm{t}-\mathrm{s})^{\alpha-1} \mathbb{E}\left\|\mathrm{x}^{\mathrm{m}}(\mathrm{s})-x^{\mathrm{O}}(\mathrm{s})\right\|^{2} \mathrm{ds} .
\end{aligned}
$$


By the well known singular version of Gronwall inequality, there exists a constant $\mathrm{K}^{*}(\alpha)$ independent of $u, m$ and $t$ such that

$$
\begin{aligned}
\mathbb{E}\left\|x^{\mathrm{m}}(\mathrm{t})-x^{0}(\mathrm{t})\right\|^{2} \leq & \mathrm{K}^{*}(\alpha)\left(\int_{0}^{\tau}\left\|\mathrm{B}(\mathrm{s}) \mathrm{u}^{\mathrm{m}}(\mathrm{s})-\mathrm{B}(\mathrm{s}) \mathrm{u}^{0}(\mathrm{~s})\right\|^{\mathrm{p}} \mathrm{ds}\right)^{\frac{2}{p}} \\
& \leq \mathrm{K}^{*}(\alpha)\left\|B u^{\mathrm{m}}-\mathrm{Bu}\right\|_{\mathrm{L}^{\mathrm{p}}([0, \tau] ; \mathrm{u})}^{2} .
\end{aligned}
$$

Since B is strongly continuous, we get

$$
\left\|\mathrm{Bu}^{\mathrm{m}}-\mathrm{Bu}^{0}\right\|_{\mathrm{L}([0, \tau] ; \mathrm{U})}^{2} \stackrel{\mathrm{s}}{\rightarrow} 0 \text { as } \mathrm{m} \rightarrow \infty .
$$

From (4.1) and (4.2), we conclude that

$$
\mathbb{E}\left\|x^{m}(t)-x^{0}(t)\right\|^{2} \rightarrow 0 \text { as } m \rightarrow \infty
$$

This implies that $\mathbb{E}\left\|x^{m}-x^{0}\right\|^{2} \rightarrow 0$ in $C\left([0, \tau] ; L^{2}(\Omega, H)\right)$ as $m \rightarrow \infty$.

Note that [HL] implies the assumptions of Balder (see Theorem 2.1, 3]). Hence, by Balder's theorem, we can conclude that

$$
(x, u) \rightarrow \mathbb{E} \int_{0}^{\tau} L(t, x(t), u(t)) d t
$$

is sequentially lower semicontinuous in the strong topology of $\mathrm{L}^{1}([0, \tau] ; \mathrm{H})$ and weak topology of $\mathrm{L}^{\mathfrak{p}}([0, \tau] ; \mathrm{U}) \subset \mathrm{L}^{1}([0, \tau] ; \mathrm{U})$. Hence, $\mathrm{J}$ is weakly lower semicontinuous on $\mathrm{L}^{\mathfrak{p}}([0, \tau] ; \mathrm{U})$, and since by $[\mathrm{HL}]$ (iv), $\mathrm{J}>-\infty, \mathrm{J}$ attains its infimum at $\mathrm{u}^{0} \in \mathrm{U}_{\mathrm{ad}}$, that is,

$$
\begin{aligned}
\varepsilon & :=\lim _{m \rightarrow \infty} \mathbb{E} \int_{0}^{\tau} \ell\left(t, x^{m}(t), u^{m}(t)\right) d t \\
& \geq \mathbb{E} \int_{0}^{\tau} \ell\left(t, x^{0}(t), u^{0}(t)\right) d t=J\left(x^{0}, u^{0}\right) \geq \varepsilon .
\end{aligned}
$$

This completes the proof.

\section{Applications}

Let $\Omega_{1} \subset \mathbb{R}^{3}$ be a bounded domain and $\partial \Omega_{1} \in \mathbb{C}^{3}$. Further let $H=U:=\mathrm{L}^{2}\left(\Omega_{1}\right), \omega(\mathrm{t})$ is a standard cylindrical Wiener process in $\mathrm{H}$ defined on a stochastic space $(\Omega, \mathcal{F}, \mathbf{P})$. Suppose $\mathrm{D}(A):=\mathrm{H}^{2}\left(\Omega_{1}\right) \cap \mathrm{H}_{0}^{1}\left(\Omega_{1}\right)$ and for $z \in \mathrm{D}(A), A z:=\left(\frac{\partial^{2}}{\partial z_{1}^{2}}+\frac{\partial^{2}}{\partial z_{2}^{2}}+\frac{\partial^{2}}{\partial z_{3}^{2}}\right) z$. The admissible control set $\mathrm{U}_{\mathrm{ad}}:=\left\{\mathfrak{u} \in \mathrm{U}:\|\mathfrak{u}\|_{\mathrm{L}^{\mathrm{p}}([0,1]: \mathrm{U})} \leq 1\right\}$.

Consider the following fractional stochastic equation

$$
\left\{\begin{array}{l}
{ }^{C} D_{t}^{\frac{2}{3}} x(t, z)=\Delta x(t, z)+\int_{0}^{1} \mathcal{K}(z, s) u(s, t) d s+\int_{0}^{1} v(z, s) \sin (x, s) d s \\
+\frac{(x(t, z))^{2}}{1+(x(t, z))^{2}} d \omega(t) \\
x(0, z)=x_{0}(z), \quad z \in \Omega_{1}, \\
\left.x(t, z)\right|_{z \in \partial \Omega}=0, \quad t>0
\end{array}\right.
$$


Define $x(t)(z)=x(t, z),(B u)(t)(z)=\int_{0}^{1} \mathcal{K}(z, s) u(s, t) d s, f(t, x(t))(z)=f(t, x(t, z))=\int_{0}^{1} v(z, s) \sin (x, s) d s$, $g(t, x(t))(z)=g(t, x(t, z))=\frac{(x(t, z))^{2}}{1+(x(t, z))^{2}}$, and $x(0)(z)=x(0, z)=x_{0}(z)$. Moreover, we assume that $\mathcal{K}: \Omega_{1} \times[0,1] \rightarrow \mathbb{R}$ is continuous. The function $v$ is measurable and $\int_{\Omega_{1}} \int_{0}^{1} v(z, s) \mathrm{d} s \mathrm{~d} z<\infty$. The one-dimensional standard Brownian motion is denoted by $\omega(t)$. Thus, for $\alpha=2 / 3$ the problem (5.1) can be written as the abstract form of system (2.2) with the cost function

$$
J(x, u):=\mathbb{E}\left\{\int_{0}^{1} \ell(t, x(t), u(t)) d t\right\}
$$

where $\ell(t, x(t), u(t))(z)=\int_{\Omega_{1}}|x(t, z)|^{2} d z+\int_{\Omega_{1}}|u(t, z)|^{2} d z$. It is easy to see that the assumptions $[\mathrm{H} 0]-[\mathrm{H} 4]$ are satisfied. If [HL] is satisfied, then there exists an optimal pair $\left(x^{0}, u^{0}\right) \in \mathrm{L}^{2}([0,1] \times$ $\left.\Omega_{1}\right) \times \mathrm{L}^{2}\left([0,1] \times \Omega_{1}\right)$ such that $\mathrm{J}\left(\mathrm{x}^{0}, \mathrm{u}^{0}\right) \leq \mathrm{J}(\mathrm{x}, \mathrm{u})$ for all $(x, \mathrm{u}) \in \mathrm{L}^{2}\left([0,1] \times \Omega_{1}\right) \times \mathrm{L}^{2}\left([0,1] \times \Omega_{1}\right)$.

\section{Conclusions}

This paper considers fractional optimal control of stochastic semilinear dynamic systems with the Caputo fractional derivative in a Hilbert space. The existence and uniqueness of mild solution are studied using the fixed point technique. It is also shown that, under some natural assumptions the Lagrange problem admits at least one optimal pair of state-control.

\section{References}

[1] Agrawal, O.P.: A general formulation and solution scheme for fractional optimal control problems. Nonlinear Dyn. 38, 323-337 (2004)

[2] Balasubramaniam, P., Tamilalagan, P.: The solvability and optimal controls for impulsive fractional stochastic integro-differential equations via resolvent operators. J. Optim. Theory Appl. 174, 139-155 (2017)

[3] Balder, E.: Necessary and sufficient conditions for $\mathrm{L}_{1}$-strong-weak lower semicontinuity of integral functional. Nonlinear Anal. TMA 11, 1399-1404 (1987)

[4] Da Prato, G., Zabczyk, J.: Stochastic Equations in Infinite Dimensions. Cambridge University Press, Cambridge (1992)

[5] Das, S.: Functional Fractional Calculus. Springer-Verlag, Berlin, Heidelberg, (2011)

[6] Erdélyi, A., Magnus, W., Oberhettinger, F., Tricomi, F.G.: Higher Transcendental Functions. Vol. 3, McGraw-Hill, New York (1955)

[7] Fitt, A.D., Goodwin, A.R.H., Wakeham, W.A.: A fractional differential equation for a MEMS viscometer used in the oil industry. J. Comput. Appl. Math. 229, 373-381 (2009) 
[8] Glockle, W.G., Nonnenmacher, T.F.: A fractional calculus approach of self-similar protein dynamics. Biophys. J. 68, 46-53 (1995)

[9] Hilfer, R.: Applications of Fractional Calculus in Physics. World Scientific, Singapore, (2000)

[10] Kilbas, A.A., Srivastava, H.M., Trujillo, J.J.: Theory and Applications of Fractional Differential Equations. Elsevier, Amsterdam (2006)

[11] Li, X., Liu, Z.: The solvability and optimal controls of impulsive fractional semilinear differential equations. Taiwanese J. Math. 19, 433-453 (2015)

[12] Marle, C.M.: Measures et Probabilités. Hermann, Paris (1974)

[13] Oldham, K.B., Spanier, J.: The Fractional Calculus,Theory and Applications of Differentiation and Integration to Arbitrary Order. Academic Press, New York (1974)

[14] Pan, X., Li, X., Zhao, J.: Solvability and optimal controls of semilinear Riemann-Liouville fractional differential equations. Abstr. Appl. Anal. Volume 2014, Article ID 216919, 11 pages, (2014)

[15] Pedjeu, J.C., Ladde, G.S.: Stochastic fractional differential equations: Modeling, method and analysis. Chaos Solitons Fract. 45, 279-293 (2012)

[16] Sakthivel, R., Ren, Y., Debbouche, A., Mahmudov, N.I.: Approximate controllability of fractional stochastic differential inclusions with nonlocal conditions. Applicable Analysis 95(11), 2361-2382 (2016)

[17] Shu, X.B., Lai, Y., Chen, Y.: The existence of mild solutions for impulsive fractional partial differential equations. Nonlinear Anal. 74, 2003-2011 (2011)

[18] Tamilalagan, P., Balasubramaniam, P.: The solvability and optimal controls for fractional stochastic differential equations driven by poisson jumps via resolvent operators. Appl. Math. Optim. DOI 10.1007/s00245-016-9380-2

[19] Wang, J.R., Wei, W., Yang, Y.L.: Fractional nonlocal integrodifferential equations of mixed type with time-varying generating operators and optimal control. Opuscula Math. 30(2), 217-234 (2010)

[20] Wang, J.R., Wei, W., Zhou, Y.: Fractional finite time delay evolution systems and optimal controls in infinite-dimensional spaces. J. Dyn. Control Syst. 17(4), 515-535 (2011)

[21] Wang, J.R., Zhou, Y.: A class of fractional evolution equations and optimal controls. Nonlinear Anal. RWA 12, 262-272 (2011)

[22] Wang, J.R., Zhou, Y.: Analysis of nonlinear fractional control systems in Banach spaces. Nonlinear Anal. TMA 74, 5929-5942 (2011) 
[23] Wang, J.R., Zhou, Y., Medved, M.: On the solvability and optimal controls of fractional integrodifferential evolution systems with infinite delay. J. Optim. Theory Appl. 152, 31-50 $(2012)$

[24] Wang, J.R., Zhou, Y., Wei, W.: A class of fractional delay nonlinear integrodifferential controlled systems in Banach spaces. Commun. Nonlinear Sci. Numer. Simulat. 16, 4049-4059 (2011)

[25] Yan, Z., Lu, F.: Existence of an optimal control for fractional stochastic partial neutral integro-differential equations with infinite delay. J. Nonlinear Sci. Appl. 8, 557-577 (2015) 